\title{
High-grade hemorrhoids requiring surgical treatment are common after laparoscopic ventral mesh rectopexy
}

\author{
J. J. van Iersel ${ }^{1,2} \cdot$ H. A. Formijne Jonkers ${ }^{1}$ - P. M. Verheijen ${ }^{1}$ - W. A. Draaisma ${ }^{1}$ • \\ E. C. J. Consten ${ }^{1}$ I. A. M. J. Broeders ${ }^{1,2}$
}

Received: 13 November 2015/ Accepted: 28 December 2015/Published online: 16 February 2016

(C) The Author(s) 2016. This article is published with open access at Springerlink.com

\begin{abstract}
Purpose To describe patients developing grade III and IV hemorrhoids requiring surgery after laparoscopic ventral mesh rectopexy (LVMR) and to explore the relationship between developing such hemorrhoids and recurrence of rectal prolapse after LVMR.

Methods All consecutive patients receiving LVMR at the Meander Medical Centre, Amersfoort, the Netherlands, between 2004 and 2013 were analyzed. Kaplan-Meier estimates were calculated for recurrences.

Results A total of 420 patients underwent LVMR. Sixtyfive of these patients (actuarial 5-year incidence 24.3, $95 \%$ confidence interval (CI) 18.6-30.0) developed symptomatic grade III/IV hemorrhoids requiring stapled or excisional hemorrhoidectomy. Re-do surgery for recurrent grade III/IV hemorrhoids was required for 15 of the 65 patients (actuarial 5-year recurrence rate 40.6, $95 \%$ CI 23.2-58.0) after the primary hemorrhoidectomy. Three of the 65 patients developed an external rectal prolapse (ERP) recurrence and eight an internal rectal prolapse (IRP) recurrence. This generated a 5-year recurrence rate of $25.3 \%$ (95\% CI 0-53.9) for ERP recurrence and $24.4 \%$ (95\% CI 9.1-39.7) for IRP recurrence. The rest of the LVMR cohort not receiving additional surgery for hemorrhoids $(n=355)$ showed significantly lower actuarial 5-year ERP $(0.8 \%, p=0.011)$ and IRP $(11 \%, p=0.020)$ recurrence rates.
\end{abstract}

\section{J. J. van Iersel \\ jj.vaniersel@meandermc.nl \\ I. A. M. J. Broeders \\ IAMJ.Broeders@meandermc.nl}

1 Department of Surgery, Meander Medical Centre, Maatweg 3, 3813 TZ Amersfoort, The Netherlands

2 Institute of Technical Medicine, MIRA, Twente University, Enschede, The Netherlands
Conclusion High-grade hemorrhoids requiring surgery may be common after LVMR. The development of highgrade hemorrhoids after LVMR might be considered a predictor of rectal prolapse recurrence.

Keywords Hemorrhoids - Hemorrhoidectomy · Laparoscopic ventral mesh rectopexy · Rectal prolapse . Recurrence

\section{Introduction}

Disorders of the pelvic floor, including urinary and fecal incontinence, pelvic organ prolapse, obstructed defecation and chronic pelvic pain, are socially disabling conditions. In the Western world, this pathology is common, affecting more than $40 \%$ of the middle-aged and older women, with a lifetime risk of undergoing surgery of $10-20 \%[1,2]$. The rectum is often involved in this multi-organ problem [3]. Various conditions including rectoceles, internal and external rectal prolapse may cause fecal incontinence and the obstructed defecation syndrome (ODS) [4].

Laparoscopic ventral mesh rectopexy (LVMR) is increasingly applied for the treatment of external rectal prolapse (ERP) and internal rectal prolapse (IRP). This technique proved to be safe and effective in terms of functional results in large cohorts of patients [5-8]. For prolapse surgery, the recurrence rate is a key indicator of success. ERP recurrence is easily assessed, but diagnosing IRP recurrence remains challenging. One hypothesis is that IRP could be the cause of high-grade hemorrhoids (III and IV), but the development of such hemorrhoids after LVMR is not well known [9]. In the past 25 years, the incidence of high-grade hemorrhoids has been quoted as up to $18 \%$ after different types of rectopexy [10-25], but specific 
literature regarding LVMR is scarce. Only a handful of relatively small series mention the incidence [5, 26-28], but none of these discuss the issue in depth. The aim of this study, therefore, was to identify patients developing highgrade hemorrhoids requiring surgical treatment after LVMR and to explore the relationship between such hemorrhoids and the recurrence of rectal prolapse following LVMR.

\section{Materials and methods}

\section{Study design}

This observational cohort study was a retrospective analysis of a prospectively maintained database and was undertaken in a large teaching hospital in the Netherlands. All consecutive patients undergoing LVMR for rectal prolapse syndromes (Table 1) between March 2004 and May 2013 were analyzed.

\section{Patients and evaluation}

Postoperatively, all patients were prescribed a laxative (Macrogol 3350/electrolytes, Movicolon ${ }^{\circledR}$, Norgine Limited, Mid Glamorgan, UK). Follow-up after LVMR was carried out according to a standardized protocol and performed at 6 weeks after surgery by one of the three participating experienced pelvic floor surgeons (P.V., E.C. and I.B.). At the 6-week follow-up, the presence of hemorrhoids, recurrence of rectal prolapse, incontinence and constipation was assessed. All patients were asked to return in the event of anorectal complaints. Patients were examined for hemorrhoids in the standing and lithotomy position using leg supports, both in rest and during straining. In addition, proctoscopy was performed. Hemorrhoids were graded using the Goligher classification [29]. Patients with grade II and III hemorrhoids were treated with rubber band ligation (RBL) first. Persisting symptomatic grade III/IV hemorrhoids ('high grade') were considered an indication for surgery, but results of LVMR were awaited for at least 10 weeks. Lower grades of hemorrhoids were not operated on. ERP recurrence was clinically assessed. IRP recurrence was defined as Oxford rectal prolapse grade III/IV IRP with symptoms of obstructed defecation or fecal incontinence. Most of these patients had a coexisting rectocele or enterocele. A dynamic MRI of the pelvic floor was done on all patients suspected of an IRP recurrence. A large part of the study cohort (most patients operated from 2004 to 2011) had participated in a previous study about the outcomes of LVMR, and therefore, a longer follow-up period was available for these patients [7]. For those patients not

Table 1 Patient characteristics, medical history and initial indications for LVMR

\begin{tabular}{lll}
\hline Patient characteristics & LVMR $N=420(\%)$ & Hemorrhoidectomy group $N=65(\%)$ \\
\hline Woman/men [mean age] & $404 / 16[61.8]$ & $61 / 4[60.4]$ \\
History & & $2.6(0-5)[12]^{\mathrm{b}}$ \\
Mean para (range) [episiotomy] & $139(33.1)[37]^{\mathrm{a}}$ & $47(72.3)$ \\
Hysterectomy & $39(9.3)$ & $4(6.2)$ \\
Cystopexy & $56(13.3)$ & $13(20.0)$ \\
Anterior colporrhaphy & $6(1.4)$ & 0 \\
Sphincter operation & $137(32.6)$ & $23(35.4)$ \\
Other abdominal surgery & $28(6.7)[7]^{\mathrm{c}}$ & $3(3.1)[2]^{\mathrm{d}}$ \\
Rubber band ligation before LVMR [second session] & $20(4.8)$ & $2(3.1)$ \\
Pre-hemorrhoidectomy-before initial LVMR & $39(9.3)[13]$ & $4(6.2)[1]$ \\
RBL between LVMR and hemorrhoidectomy [second RBL] & & $5(7.7)$ \\
Indication for initial LVMR & $55(13.1)$ & $44(67.7)$ \\
ERP & $266(63.3)$ & $16(24.6)$ \\
IRP and/or symptomatic rectocele & $99(23.6)$ & \\
IRP and/or symptomatic rectocele with enterocele & & \\
\hline
\end{tabular}

$L V M R$ laparoscopic ventral mesh rectopexy, ERP external rectal prolapse, $I R P$ internal rectal prolapse, $R B L$ rubber band ligation

${ }^{\text {a }}$ In 25 patients

b In 7 patients

c Two patients underwent a third and a fourth session

${ }^{\mathrm{d}}$ One patient underwent a third and a fourth session

e Oxford rectal prolapse grade III/IV 
included in this previous study, no additional effort was made to systematically follow them up. Kaplan-Meier curves were used to establish whether there was a difference in outcome between the two groups.

\section{Surgical technique}

All laparoscopic ventral mesh rectopexies were performed according to the technique described by D'Hoore et al. [6]. All meshes used were synthetic. Either a stapled hemorrhoidectomy (SH) or a traditional excisional hemorrhoidectomy (TEH) was performed. Where a SH was not technically possible, a TEH was done. Surgery was performed by, or under direct supervision of, one of the three pelvic floor surgeons (P.V., E.C. and I.B.). Operations were performed under general or spinal anesthesia. The patients were placed in the lithotomy position using adaptive leg supports with swing stirrups. The PPH 03 stapler produced by Ethicon (EndoSurgery, Cincinnati, Ohio, USA) was used for SH. The stapled procedure had been previously standardized and was performed according to the technique described by Singer et al. [30]. Excisional hemorrhoidectomy was performed according to standard protocol [31].

\section{Statistical analysis}

Statistical Package for the Social Science Advanced version 20.0 (IBM Corp., Armonk, NY, USA) was used for statistical analysis. Data are presented as percentage, median and range. Because of differences in follow-up between patients, the Kaplan-Meier method was used to estimate the incidence of postoperative high-grade hemorrhoids and recurrence rates at various points in time. The risk estimates after a period of 5 years are shown in the text. $p<0.05$ was considered statistically significant.

\section{Results}

\section{Patients characteristics}

Before LVMR, twenty-eight patients $(6.7 \%)$ underwent RBL for grade II hemorrhoids and 20 patients $(4.8 \%)$ underwent a hemorrhoidectomy for grade III/IV hemorrhoids.

A total of 420 patients ( 16 men; 404 women) underwent LVMR. Indications for surgery were ERP $(n=55$, $13.1 \%$ ), IRP (Oxford rectal prolapse grade III/IV) and/or symptomatic rectocele $(n=266,63.3 \%)$ and IRP and/or symptomatic rectocele combined with enterocele $(n=99$, $23.6 \%)$. General patient characteristics are presented in Table 1.

\section{Follow-up}

The median follow-up after LVMR was 16.0 months (range 0.4-93.7). Three hundred and ninety-one patients $(93.1 \%)$ were available for follow-up after the standardized outpatient visit at 6 weeks postoperatively. Nine patients $(2.1 \%)$ died of causes unrelated to the LVMR within the study period.

During follow-up after LVMR, 89 patients required treatment for hemorrhoids, of which 24 were treated sufficiently by RBL. The remaining 65 patients (KaplanMeier estimate of $24.3 \%$ at 5 years, $95 \%$ CI 18.6-30.0, Table 2) received surgical treatment for symptomatic grade III/IV hemorrhoids and are referred to as the 'hemorrhoidectomy group' (63 SH, $2 \mathrm{TEH}$ ). Four of the hemorrhoidectomy group $(6.2 \%)$ received RBL between LVMR and the hemorrhoidectomy without sufficient result (flowchart Fig. 1). The median duration between LVMR and hemorrhoid surgery was 6.2 months (2.5-45.3).

\section{High-grade hemorrhoids recurrence}

Fifteen patients of the hemorrhoidectomy group (15/65) needed re-do surgery $(n=13 \mathrm{SH})$ for recurrent grade III/ IV hemorrhoids after a median of 8.3 months (1.5-40.5) after the primary hemorrhoidectomy. The estimated percentages (Kaplan-Meier) were $31.2 \%$ after $1,35.2 \%$ after 3 and $40.6 \%$ after 5 years (95\% CI 23.2-58.0, Table 2). One patient received an excisional hemorrhoidectomy after twice a SH in a period of 8.9 months. This was the only patient receiving more than two hemorrhoidectomies after LVMR.

\section{Rectal prolapse recurrence-ERP}

In the hemorrhoidectomy group, three patients (3/65) developed a clinical full-thickness external prolapse generating a recurrence percentage (Kaplan-Meier estimates) $0 \%$ after $1,2.0 \%$ after 3 and $25.3 \%$ after 5 years $(95 \%$ CI 0-53.9). Two of these patients underwent re-do LVMR and the third patient declined surgery. The ERP recurrence rate (Kaplan-Meier estimates) in the group of patients who did not received additional surgery for hemorrhoids after LVMR ('non-hemorrhoidectomy group,' $n=355$ ) was $0.8 \%$ after 5 years (95\% CI $0-2.0)$. This is significantly ( $p=0.011)$ lower compared to the hemorrhoidectomy group ( $n=65$, Fig. 2a and Table 2).

\section{Rectal prolapse recurrence-IRP}

Additionally, eight patients of the hemorrhoidectomy group (8/65) were diagnosed with an IRP recurrence. The Kaplan-Meier estimates for IRP recurrence were 1.9, 20.2 
Table 2 Kaplan-Meier estimates (\%) for incidence and recurrence of gr. III/IV hemorrhoids and recurrence of rectal prolapse in the hemorrhoidectomy group $(n=65)$ and the non-hemorrhoidectomy group $(n=355)$ at various time points

\begin{tabular}{|c|c|c|c|}
\hline \multirow[t]{2}{*}{ Kaplan-Meier estimates $\%[\mathrm{CI}]$} & \multicolumn{3}{|l|}{ Years } \\
\hline & 1 & 3 & 5 \\
\hline Gr. III/IV hemorrhoids after LVMR & 16.5 [CI 12.4-20.6] & 22.2 [CI 17.1-27.3] & 24.3 [CI 18.6-30.0] \\
\hline \multicolumn{4}{|l|}{ Recurrence high-grade hemorrhoids } \\
\hline Hemorrhoidectomy group $(n=65)$ & 31.2 [CI 16.9-45.5] & 35.2 [CI 19.7-50.7] & 40.6 [CI 23.2-58.0] \\
\hline \multicolumn{4}{|l|}{ External rectal prolapse recurrence } \\
\hline Hemorrhoidectomy group $(n=65)$ & 0 & 2.0 [CI $0-5.9]$ & 25.3 [CI 0-53.9] \\
\hline Non-hemorrhoidectomy group $(n=355)^{\mathrm{a}}$ & 0.8 [CI $0-2.0]$ & 0.8 [CI $0-2.0]$ & $0.8^{\mathrm{b}}[\mathrm{CI} 0-2.0]$ \\
\hline \multicolumn{4}{|l|}{ Internal rectal prolapse recurrence } \\
\hline Hemorrhoidectomy group $(n=65)$ & 1.9 [CI $0-5.6]$ & 20.2 [CI 6.5-33.9] & 24.4 [CI 9.1-39.7] \\
\hline Non-hemorrhoidectomy group $(n=355)^{\mathrm{a}}$ & $2.1[\mathrm{CI} 0.3-3.7]$ & 5.7 [CI 2.0-9.4] & 11.0 [CI 4.3-17.7] \\
\hline
\end{tabular}

CI $95 \%$ confidence interval, LVMR laparoscopic ventral mesh rectopexy, gr. grade

${ }^{\text {a }}$ This cohort contains the 420 patients receiving a LVMR minus the patients developing postoperative high-grade hemorrhoids; $420-65=355$

b One ERP recurrence after 64.6 months

Fig. 1 Flowchart. LVMR laparoscopic ventral mesh rectopexy, $R B L$ rubber band ligation, $S H$ stapled hemorrhoidectomy, TEH traditional excisional hemorrhoidectomy, $R P$ rectal prolapse, $G r$. grade. $E R P$ external rectal prolapse, IRP internal rectal prolapse. ${ }^{\mathrm{a}}$ Four of these patients received an re-SH first

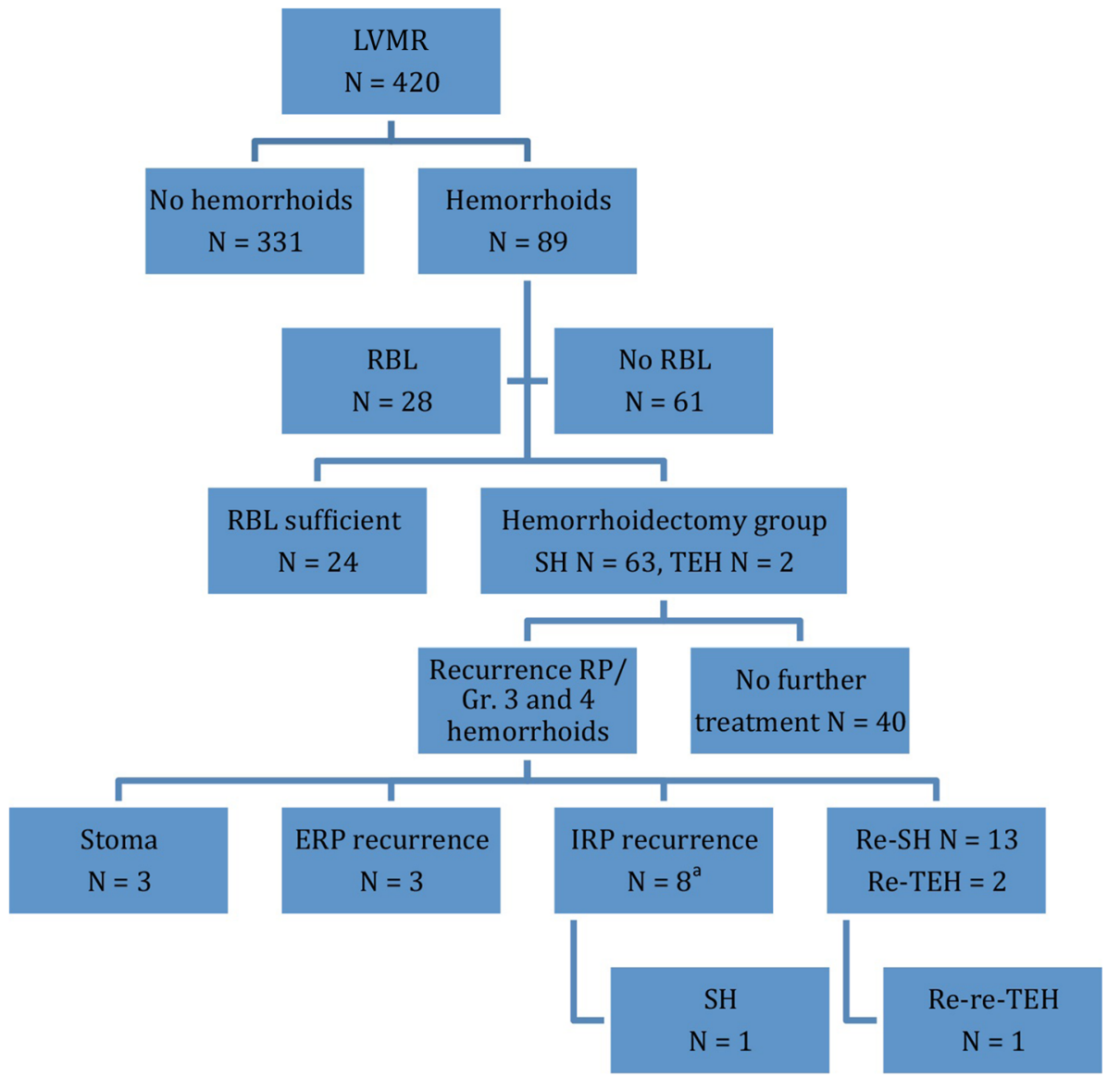

and $24.4 \%$ after 1,3 and 5 years (95\% CI 9.1-39.7). One patient decided against surgery and the rest received re-do rectopexy. One patient required a SH 9 months after the re- do rectopexy. Due to persistent fecal incontinence without curative surgical options, a stoma was created in a further three patients. 

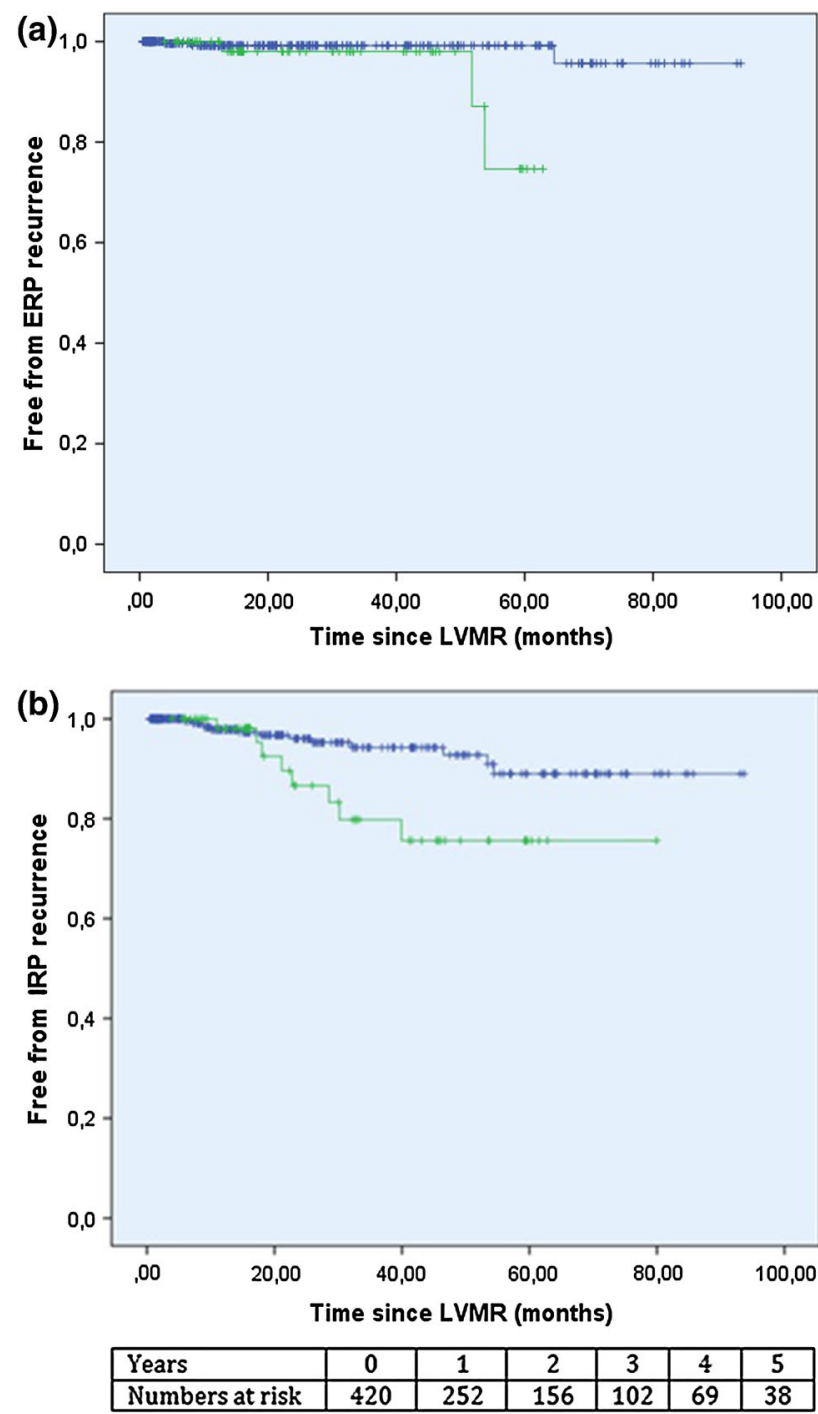

Fig. 2 a Kaplan-Meier curve for ERP recurrence (cohort $n=420$ ). The green line represents the cohort developing high-grade hemorrhoids after LVMR ('hemorrhoidectomy group,' $n=65$ ), and the blue line represents the rest of the LVMR cohort not developing highgrade hemorrhoids after LVMR ('non-hemorrhoidectomy group' $n=355, p=0.011)$. b Kaplan-Meier curve for IRP recurrence (cohort $n=420$ ). The green line represents the cohort developing high-grade hemorrhoids after LVMR ('hemorrhoidectomy group,' $n=65$ ), and the blue line represents the rest of the LVMR cohort not developing high-grade hemorrhoids after LVMR ('non-hemorrhoidectomy group,' $n=355, p=0.020$ ). The duration of eventfree survival was measured from date of LVMR to the time of the event (complete) or the last follow-up (censored) for both curves. At the bottom of the figure, a table with the number of patients left for analysis per year is presented

The estimated IRP recurrence percentage after 5 years was significantly lower $(11 \%, p=0.020,95 \%$ CI $4.3-17.7)$ in the non-hemorrhoidectomy group $(n=355)$ compared to the hemorrhoidectomy group $(n=65$, Fig. $2 \mathrm{~b}$ and Table 2).

\section{Discussion}

The exact incidence of high-grade hemorrhoids following LVMR is not known. Our study found a high actuarial 5 -year incidence of $24.3 \%$. In the literature, only four articles report on this issue, quoting a lower incidence varying from 1.6 to $5 \%$ (Table 3) [5, 26-28]. However, these studies describe the outcome of LVMR rather than focus on the development of postoperative high-grade hemorrhoids. Of the two studies with substantial follow-up, the study of D'Hoore et al. only followed up the patients by telephone and Slawik et al. did not perform an anorectal examination after 3 years [5, 27]. In the other two studies, the follow-up period was substantial shorter [26, 28]. As a result, in these studies the occurrence of high-grade hemorrhoids after LVMR may have been underestimated. Furthermore, in the reported studies the main indication for LVMR was ERP, whereas in our study $86.9 \%$ of the patients presented with IRP. Also, two studies combined the LVMR with other procedures (e.g., STARR or resection rectopexy) [27, 28]. This heterogeneity among studies might explain the differences in reported percentages of high-grade hemorrhoids after LVMR. In our study, $53.8 \%$ of the hemorrhoidectomy group (35/65) suffered from ODS complaints before LVMR and most of them had a long history of straining and incomplete evacuation. After LVMR, still 15 patients of the hemorrhoidectomy group $(15 / 65,23.1 \% . p=0.004)$ reported persisting ODS complaints. Other studies quote a slightly lower figure (up to $19 \%$ ) of patients suffering from persisting ODS after LVMR [5-8]. The high incidence of grade III/IV hemorrhoids after LVMR and the high recurrence rate of grade III/IV hemorrhoids after hemorrhoidectomy might be the result of persistent straining. The actuarial 5-year recurrence rate of $40.6 \%$ for grade III/IV hemorrhoids after hemorrhoidectomy was very high compared to several randomized controlled trials showing recurrence rates from 0 to $5 \%$ for both excisional as stapled hemorrhoidectomy [32-35]. It is also possible that some of the patients in the hemorrhoidectomy group were not properly assessed before LVMR. Possibly, some patients underwent LVMR for symptomatic IRP combined with a rectocele, whereas retrospectively the symptoms might have been caused mostly by a mucosal prolapse. Consequently, it could be that high-grade hemorrhoids following LVMR might be attributed to residual mucosal prolapse in some cases. In these patients, it seems that LVMR repairs the rectal prolapse, but fails to correct the mucosal prolapse. If so, a different operation instead of LVMR (e.g., STARR) might have been more appropriate. Because of the retrospective character of this study, it is unfortunately not possible to verify if mucosal prolapses were missed before LVMR. 
Table 3 Available literature concerning high-grade hemorrhoids requiring surgery after LVMR

\begin{tabular}{|c|c|c|c|c|c|}
\hline First author & No. of patients & $\begin{array}{l}\text { Indication } \\
\text { LVMR }\end{array}$ & $\begin{array}{l}\text { Follow-up in } \\
\text { months (median) }\end{array}$ & $\begin{array}{l}\text { High-grade hemorrhoids } \\
\text { after LVMR }\end{array}$ & Treatment \\
\hline D’Hoore [5] & 42 & ERP & 61 & $1(2.4 \%)$ & $\mathrm{SH}$ \\
\hline Slawik $^{\mathrm{a}}$ [27] & 80 & $\begin{array}{l}44 \text { ERP } \\
36 \text { IRP }\end{array}$ & 54 & $4(5 \%)$ & $3 \mathrm{SH}, 1 \mathrm{TEH}$ \\
\hline Wijffels [26] & 80 & ERP & 23 & $2(2.5 \%)$ & $1 \mathrm{SH}, 1 \mathrm{STARR}$ \\
\hline Randall $^{\mathrm{b}}[28]$ & 190 & ERP & 29 & $3(1.6 \%)$ & $3 \mathrm{SH}$ \\
\hline
\end{tabular}

LVMR laparoscopic ventral mesh rectopexy, SH stapled hemorrhoidectomy, STARR stapled transanal rectal resection

${ }^{a}$ Seven patients underwent a laparoscopic resection rectopexy, and 74 females underwent concurrent posterior colporrhaphy and vaginal sacrocolpopexy

${ }^{\mathrm{b}}$ LVMR was combined with Orr-Loygue $(n=3)$, anterior colporrhaphy $(n=7)$, posterior STARR $(n=10)$ and SH $(n=2)$

The role of mucosal prolapse in hemorrhoidal disease is in debate. Gaj et al. [36] showed that $40 \%$ of the proctologists do not consider mucosal prolapse as independent from hemorrhoidal disease in a national survey. We believe that mucosal prolapse is an integral part of the hemorrhoidal disease. However, whether mucosal prolapse is a completely different entity or not, with excising a circumferential band of excessive rectal mucosa and submucosa and interrupting the blood supply of the superior hemorrhoidal artery proximal to the hemorrhoidal tissue, the clinical condition is treated either way.

In addition, it is worth noting that the hemorrhoidectomy group includes more patients with a history of hysterectomy (72.3\% vs. 33.1), re-do of the initial LVMR $(12.3 \%$ vs. $8.8 \%)$ and number of past episiotomies ( $8.8 \%$ vs. $18.5 \%)$ than the non-hemorrhoidectomy group. All these variables might constitute an increased risk of developing high-grade hemorrhoids after LVMR. No other differences worth mentioning were found between the groups.

The incidence of recurrence of rectal prolapse in the hemorrhoidectomy group was also high, with an actuarial 5-year ERP recurrence rate of $25.3 \%$ and an actuarial 5 -year IRP recurrence rate of $24.4 \%$. In contrast, the nonhemorrhoidectomy group $(n=355)$ showed significantly lower actuarial 5-year ERP $(0.8 \%, p=0.011)$ and IRP $(11 \%, p=0.020)$ recurrence rates (Fig. 2a/b; Table 2). The literature quotes similar incidences to our non-hemorrhoidectomy group with rates varying from 1.6 to $4.8 \%$ $[5,6,28,37]$ for ERP and from 0 to $15 \%$ for IRP $[8,38]$. This could suggest that patients with high-grade hemorrhoids after rectopexy are susceptible to developing a rectal prolapse recurrence after LVMR. The hemorrhoidectomy group seems to contain a cohort of patients with persisting symptoms possibly not well responding to the standard therapy. Both high recurrence rates of rectal prolapse and grade III/IV hemorrhoids are indicative. The three patients requiring a stoma due to persistent fecal incontinence support this impression. It may be that high-grade hemorrhoids after LVMR are a sign of laxity of (a part of) the posterior compartment and represent the first stage of a continuum, eventually developing into rectal prolapse. Consequently, the findings of this study could suggest that the development of high-grade hemorrhoids following LVMR might be considered predictive of a rectal prolapse recurrence. In order to exclude a rectal prolapse recurrence, additional radiological imaging should be considered when a patient presents with grade III/IV hemorrhoids following LVMR. Unfortunately, our data did not offer a clear explanation for the relationship between post-LVMR highgrade hemorrhoids and rectal prolapse recurrence. As there is no literature available on this potential relationship, it would be an interesting topic for future studies.

LVMR has been performed in our hospital since 2004. Analysis shows that the occurrence of post-LVMR hemorrhoidectomy is fairly stable over the years. This indicates that there is probably no learning curve problem, or sign of insufficient repair. This is supported by the rates for ERP and IRP recurrence in the non-hemorrhoidectomy group which are comparable to the contemporary literature.

A limitation of this paper is the differences in follow-up between patients. Although the Kaplan-Meier method yields appropriate estimates for recurrence rates at various points in time, underestimation remains possible. When we compared patients receiving extended follow-up in the context of a previous study $(n=149)$ [7] with those followed up according to the standardized postoperative protocol $(n=271)$, the risk of high-grade hemorrhoids was somewhat higher with the standardized postoperative follow-up. However, estimates were unstable and the difference was not statistically significant $(p=0.149)$. In the standardized follow-up protocol, the probability of the patient presenting at the outpatient clinic after the standard 6 week postoperative control might be related to the degree of postoperative complaints, and therefore, selection bias may have occurred. 


\section{Conclusion}

High-grade hemorrhoids requiring surgery may be common after LVMR. The development of high-grade hemorrhoids after LVMR might be considered a predictor of rectal prolapse recurrence.

\section{Compliance with ethical standards}

Conflict of interest The authors declare that they have no conflict of interest.

Ethical approval All procedures performed in this study were in accordance with the ethical standards of the institutional research committee and with the 1964 Declaration of Helsinki and its later amendments.

Informed consent Formal consent is not required for this type of study.

Open Access This article is distributed under the terms of the Creative Commons Attribution 4.0 International License (http://crea tivecommons.org/licenses/by/4.0/), which permits unrestricted use, distribution, and reproduction in any medium, provided you give appropriate credit to the original author(s) and the source, provide a link to the Creative Commons license, and indicate if changes were made.

\section{References}

1. de Boer TA, Slieker-Ten Hove MCP, Burger CW, Kluivers KB, Vierhout ME (2011) The prevalence and factors associated with previous surgery for pelvic organ prolapse and/or urinary incontinence in a cross-sectional study in The Netherlands. Eur $\mathbf{J}$ Obstet Gynecol Reprod Biol 158:343-349

2. Sung VW, Hampton BS (2009) Epidemiology of pelvic floor dysfunction. Obstet Gynecol Clin North Am 36:421-443

3. Elneil S (2009) Complex pelvic floor failure and associated problems. Best Pract Res Clin Gastroenterol 23:555-573

4. Beck DE, Allen NL (2010) Rectocele. Clin Colon Rectal Surg 23:90-98

5. D'Hoore A, Cadoni R, Penninckx F (2004) Long-term outcome of laparoscopic ventral rectopexy for total rectal prolapse. Br J Surg 91:1500-1505

6. D'Hoore A, Penninckx F (2006) Laparoscopic ventral recto(colpo)pexy for rectal prolapse: surgical technique and outcome for 109 patients. Surg Endosc 20:1919-1923

7. Formijne Jonkers HA, Poierrié N, Draaisma WA, Broeders IAMJ, Consten ECJ (2013) Laparoscopic ventral rectopexy for rectal prolapse and symptomatic rectocele: an analysis of 245 consecutive patients. Color Dis 15:695-699

8. Collinson R, Wijffels N, Cunningham C, Lindsey I (2010) Laparoscopic ventral rectopexy for internal rectal prolapse: shortterm functional results. Colorectal Dis 12:97-104

9. Boccasanta P, Venturi M, Roviaro G (2007) Stapled transanal rectal resection versus stapled anopexy in the cure of hemorrhoids associated with rectal prolapse. A randomized controlled trial. Int J Colorectal Dis 22:245-251

10. Blatchford GJ, Perry RE, Thorson AG, Christensen MA (1989) Rectopexy without resection for rectal prolapse. Am J Surg 158:574-576
11. Yoshioka K, Heyen F, Keighley MR (1989) Functional results after posterior abdominal rectopexy for rectal prolapse. Dis Colon Rectum 32:835-838

12. Madoff RD, Williams JG, Wong WD, Rothenberger DA, Goldberg SM (1992) Long-term functional results of colon resection and rectopexy for overt rectal prolapse. Am J Gastroenterol 87:101-104

13. Huber FT, Stein H, Siewert JR (1995) Functional results after treatment of rectal prolapse with rectopexy and sigmoid resection. World J Surg 19:138-143 discussion 143

14. Chow PK, Ho YH (1996) Abdominal resection rectopexy versus Delorme's procedure for rectal prolapse: comparison of clinical and physiological outcomes. Int J Colorectal Dis 11:201-202

15. Poen AC, de Brauw M, Felt-Bersma RJ, de Jong D, Cuesta MA (1996) Laparoscopic rectopexy for complete rectal prolapse. Clinical outcome and anorectal function tests. Surg Endosc 10:904-908

16. Galili Y, Rabau M (1997) Comparison of polyglycolic acid and polypropylene mesh for rectopexy in the treatment of rectal prolapse. Eur J Surg 163:445-448

17. Boccasanta P, Rosati R, Venturi M et al (1998) Comparison of laparoscopic rectopexy with open technique in the treatment of complete rectal prolapse: clinical and functional results. Surg Laparosc Endosc 8:460-465

18. Zittel TT, Manncke K, Haug S et al (2000) Functional results after laparoscopic rectopexy for rectal prolapse. J Gastrointest Surg 6:632-641

19. Benoist S, Taffinder N, Gould S, Chang A, Darzi A (2001) Functional results 2 years after laparoscopic rectopexy. Am J Surg 182:168-173

20. Lechaux D, Trebuchet G, Siproudhis L, Campion JP (2005) Laparoscopic rectopexy for full-thickness rectal prolapse: a single-institution retrospective study evaluating surgical outcome. Surg Endosc 19:514-518

21. Byrne CM, Smith SR, Solomon MJ, Young JM, Eyers AA, Young CJ (2008) Long-term functional outcomes after laparoscopic and open rectopexy for the treatment of rectal prolapse. Dis Colon Rectum 51:1597-1604

22. Ashari LHS, Lumley JW, Stevenson ARL, Stitz RW (2005) Laparoscopically-assisted resection rectopexy for rectal prolapse: 10 years' experience. Dis Colon Rectum 48:982-987

23. Carpelan-Holmström M, Kruuna O, Scheinin T (2006) Laparoscopic rectal prolapse surgery combined with short hospital stay is safe in elderly and debilitated patients. Surg Endosc 20:1353-1359

24. von Papen M, Ashari LHS, Lumley JW, Stevenson ARL, Stitz RW (2007) Functional results of laparoscopic resection rectopexy for symptomatic rectal intussusception. Dis Colon Rectum 50:50-55

25. Kosba Y, Elshazly WG, Abd El Maksoud W (2010) Posterior sagittal approach for mesh rectopexy as a management of complete rectal in adults. Int J Colorectal Dis 25:881-886

26. Wijffels N, Cunningham C, Dixon A, Greenslade G, Lindsey I (2011) Laparoscopic ventral rectopexy for external rectal prolapse is safe and effective in the elderly. Does this make perineal procedures obsolete? Colorectal Dis 13:561-566

27. Slawik S, Soulsby R, Carter H, Payne H, Dixon AR (2008) Laparoscopic ventral rectopexy, posterior colporrhaphy and vaginal sacrocolpopexy for the treatment of recto-genital prolapse and mechanical outlet obstruction. Colorectal Dis 10:138-143

28. Randall J, Smyth E, McCarthy K, Dixon AR (2014) Outcome of laparoscopic ventral mesh rectopexy for external rectal prolapse. Colorectal Dis 16:914-919 
29. Clinical Practice Committee AGA (2004) American Gastroenterological Association medical position statement: diagnosis and treatment of hemorrhoids. Gastroenterology 126:1461-1462

30. Singer MA, Cintron JR, Fleshman JW et al (2002) Early experience with stapled hemorrhoidectomy in the United States. Dis Colon Rectum 45:360-367 discussion 367-369

31. Kim J-S, Vashist YK, Thieltges S et al (2013) Stapled hemorrhoidopexy versus Milligan-Morgan hemorrhoidectomy in circumferential third-degree hemorrhoids: long-term results of a randomized controlled trial. J Gastrointest Surg 17:1292-1298

32. Pavlidis T, Papaziogas B, Souparis A, Patsas A, Koutelidakis I, Papaziogas T (2002) Modern stapled Longo procedure versus conventional Milligan-Morgan hemorrhoidectomy: a randomized controlled trial. Int J Colorectal Dis 17:50-53

33. Shalaby R, Desoky A (2001) Randomized clinical trial of stapled versus Milligan-Morgan haemorrhoidectomy. $\mathrm{Br} \quad \mathrm{J} \quad$ Surg 88:1049-1053
34. Gravié JF, Lehur P-A, Huten N et al (2005) Stapled hemorrhoidopexy versus Milligan-Morgan hemorrhoidectomy: a prospective, randomized, multicenter trial with 2-year postoperative follow up. Ann Surg 242:29-35

35. Hetzer FH, Demartines N, Handschin AE, Clavien P-A (2002) Stapled versus excision hemorrhoidectomy: long-term results of a prospective randomized trial. Arch Surg 137:337-340

36. Gaj F, Trecca A (2005) Hemorrhoids and rectal internal mucosal prolapse: one or two conditions? A national survey. Tech Coloproctol 9:163-165

37. Boons P, Collinson R, Cunningham C, Lindsey I (2010) Laparoscopic ventral rectopexy for external rectal prolapse improves constipation and avoids de novo constipation. Colorectal Dis 12:526-532

38. Gouvas N, Georgiou PA, Agalianos C et al (2015) Ventral colporectopexy for overt rectal prolapse and obstructed defaecation syndrome: a systematic review. Colorectal Dis 17:O34-O46 